\title{
Pulsed Discharge Plasma in Slug-flow Reactor System for Water Pollutant Removal and Nanoparticle Synthesis
}

\author{
Wahyudiono $^{1, a}$, Siti Machmudah ${ }^{2, \mathrm{~b}}$, Hideki Kanda ${ }^{1, \mathrm{c}}$, Yaping Zhao ${ }^{3, \mathrm{~d}}$, and Motonobu Goto ${ }^{1, \mathrm{e}, *}$ \\ 1 Department of Materials Process Engineering, Nagoya University, Nagoya 464-8603, Japan \\ 2 Department of Chemical Engineering, Sepuluh Nopember Institute of Technology, Surabaya 60111, \\ Indonesia \\ 3 School of Chemistry \& Chemical Engineering, Shanghai Jiao Tong University, Shanghai 200240, China \\ E-mail: ${ }^{a} w a h y u d i o n o @ b . m b o x . n a g o y a-u . a c . j p,{ }^{b}$ machmudah@chem-eng.its.ac.id, \\ ckanda.hideki@material.nagoya-u.ac.jp, dypzhao@sjtu.edu.cn, e, ${ }^{*}$ goto.motonobu@material.nagoya-u.ac.jp \\ (Corresponding author)
}

\begin{abstract}
The water pollutants removal and the metal nanoparticles synthesis were demonstrated by the applying high voltage discharge plasma at room temperature. The experiments were performed in continuous system by the flowing liquid and gas phases simultaneously in a glass capillary tube, where various gas types were employed as a gas phase. The optical emission spectroscopy (OES) equipment identified that the reactive oxidation species were obviously observed at each gas type. The generation rate of them can be enhanced significantly by changing the reactor type from straight to helical form. The water pollutants removal rate has order from oxygen $>$ air $>$ nitrogen $>$ argon $>$ helium. Furthermore, the silver or gold nanoparticles can be synthesized by this plasma reactor system. The transmission electron microscopy (TEM) images indicated that the particle products had spherical morphology with a diameter size smaller than $10 \mathrm{~nm}$. It was deduced that the process presented here is an innovative and applicable in practice, and it can lead to advanced organic compounds degradation and nanoparticles synthesis technology.
\end{abstract}

Keywords: Discharge plasma, slug-flow reactor, water pollutant, nanoparticle.

ENGINEERING JOURNAL Volume 25 Issue 9

Received 8 May 2021

Accepted 1 September 2021

Published 30 September 2021

Online at https:/ / engj.org/

DOI:10.4186/ej.2021.25.9.1 


\section{Introduction}

Plasma, mostly, can be stated as a partially or fully ionized gas consisting of electrons, ions, and neutral species, and it can be formed by applying energy to an inert gas, resulting in the charge carrier generation. When the electron or the photon with enough energy collides with neutral molecule and atom in the input gas (photoionization or electron-impact ionization), the electron and ion in the gas state would be generated. These collisions can be either inelastic or elastic collision [1-3]. Since the interaction between plasma and water may offer the unique or favorable properties medium for the experimental chemists, their interaction has attracted much interest for the various applications $[1,2,4]$. Moreover, since the implementation in various applications is easy with cheap operating cost, the high voltage discharge plasma in liquid and gas-liquid environments at atmospheric condition become to have superior popularity.

When the high voltage discharge plasma was applied in liquid water or gas-liquid water environment, the reactive molecules and the reactive species which possess high reactivity, i.e. hydrogen peroxide molecule, oxygen molecule, hydrogen molecule, and hydroxyl radicals, might be generated. Besides the chemical phenomena, several of physical phenomena, i.e. shock wave generation, electrical field, and ultraviolet radiation, also would be found during the applying high voltage discharge plasma [5-7]. Since, in a gas medium, the discharge plasma normally is more easily to be initiated than in a liquid medium, the less energy was needed to generate plasma in a gas-liquid media than that was needed to generate plasma that was directly introduced in a liquid medium [8-10]. This is, of course, a positive effect in terms of operating cost during the applying high voltage discharge plasma. Here, the high voltage discharge plasma in a gas-liquid media was utilized to decompose water pollutant compounds $[11,12]$ and to synthesize nanoparticles $[13,14]$ in a slug-flow reactor system with argon gas as the gas phase.

In this manuscript, we presented the review of our previous related studies where methylene blue (MB) and coomassie brilliant blue $\mathrm{R}-250$ (CBB) were selected as water pollutants model compounds [11,12]. These organic dye compounds possessed an intense color and have been used widely in industries such as printing, textile, and pharmaceutical [15-17]. Their use may generate the wastewater containing organic dye compounds in the environment in a large volume. Although at a low concentration, the existence of these dye compounds in wastewater can result in water pollutants. Moreover, $\mathrm{MB}$ and $\mathrm{CBB}$ were also ordinary employed to represent organic dye compounds that are very hard to decompose in wastewater streams by using the irradiation of visible-light process. In addition to the decomposition of water pollutant compounds, this high voltage discharge plasma system was also employed to synthesize silver and gold nanoparticles [13, 14]. These nanoparticles were known to have distinctive properties, i.e. optical absorption spectra, biological, easy surface modification, high electrical conductivity, and thermal properties; Hence, they can be involved in diverse applications [18-20]. In subsequent, the high voltage discharge plasma in this system is able to enhance the decomposition rate of water pollutant compounds and to improve the metal nanoparticle production rate.

\section{Plasma as a Reaction Medium}

As informed above, in the discharge plasma system with liquid water or gas-liquid water environment, the high level and potential energy of radical, ion, and excited species were found and may serve as highly reactive media for various chemical processes. During applying the high voltage discharge plasma in an aqueous media, the reactions occurring can be presumed to be as follows $[11,21,22]$. The reactions were initiated by the dissociation of water and ionisation of water molecule.

$$
\begin{aligned}
& \mathrm{H}_{2} \mathrm{O}+\mathrm{e}^{-} \rightarrow \mathrm{H}_{2} \mathrm{O}^{*}+\mathrm{e}^{-} \\
& \mathrm{H}_{2} \mathrm{O}+\mathrm{e}^{-} \rightarrow \mathrm{OH}^{\bullet}+\mathrm{H}^{\bullet}+e^{-} \\
& \mathrm{H}_{2} \mathrm{O} \rightarrow \mathrm{H}_{2}+\frac{1}{2} \mathrm{O}_{2} \\
& \mathrm{H}_{2} \mathrm{O}+\mathrm{e}^{-} \rightarrow \mathrm{H}_{2} \mathrm{O}^{+}+2 \mathrm{e}^{-} \\
& \mathrm{H}_{2} \mathrm{O}^{+}+\mathrm{H}_{2} \mathrm{O} \rightarrow \mathrm{H}_{3} \mathrm{O}^{+}+\mathrm{OH}
\end{aligned}
$$

The formed radicals may interact with each other to generate stable hydrogen molecule, hydrogen peroxide molecule, or regenerated water.

$$
\begin{aligned}
& \mathrm{H}^{\bullet}+\mathrm{H}^{\bullet} \rightarrow \mathrm{H}_{2} \\
& \mathrm{OH}^{\bullet}+\mathrm{OH}^{\bullet} \rightarrow \mathrm{H}_{2} \mathrm{O}_{2} \\
& \mathrm{H}^{\bullet}+\mathrm{OH}^{\bullet} \rightarrow \mathrm{H}_{2} \mathrm{O}
\end{aligned}
$$

To favor this condition, the high voltage discharge plasma was applied in a slug-flow reactor system with diverse gases, i.e. nitrogen $\left(\mathrm{N}_{2}\right)$, oxygen $\left(\mathrm{O}_{2}\right)$, helium $(\mathrm{He})$, and argon (Ar), as the gas phase. Fig. 1 illustrated the schematic diagram apparatus for discharge plasma generation in a slug-flow reactor system. This apparatus consists of a glass capillary tube as a slug-flow reactor that has $2.0 \mathrm{~mm}$ inner diameter; the HPLC pump (LC-10AD, Shimadzu Co., Japan), to pump the starting materials; the gas flow meter (RK-1250, Kofloc Instruments Inc., Japan), to adjust the gas phase flow rate; and the pulsed power supply (TE-HVP1510K300-NP, Tamaoki Electronics Co. Ltd., Japan), to generate electrical discharge plasma. The procedure experiment was described elsewhere [11-14]. To form the gas-liquid slug-flow condition, the gas and the liquid from two 
different streams were flowed in the glass capillary tube simultaneously. They were met via the union Teejunction (SS-200-3, Swagelok).

\section{(a). Straight type reactor.}

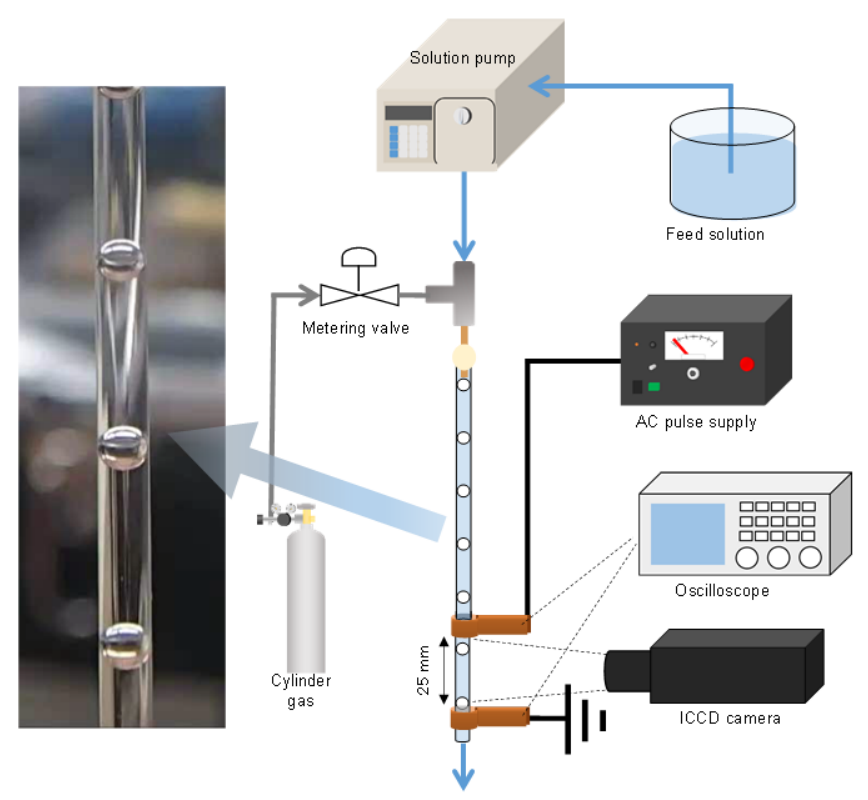

(b). Helical type reactor.

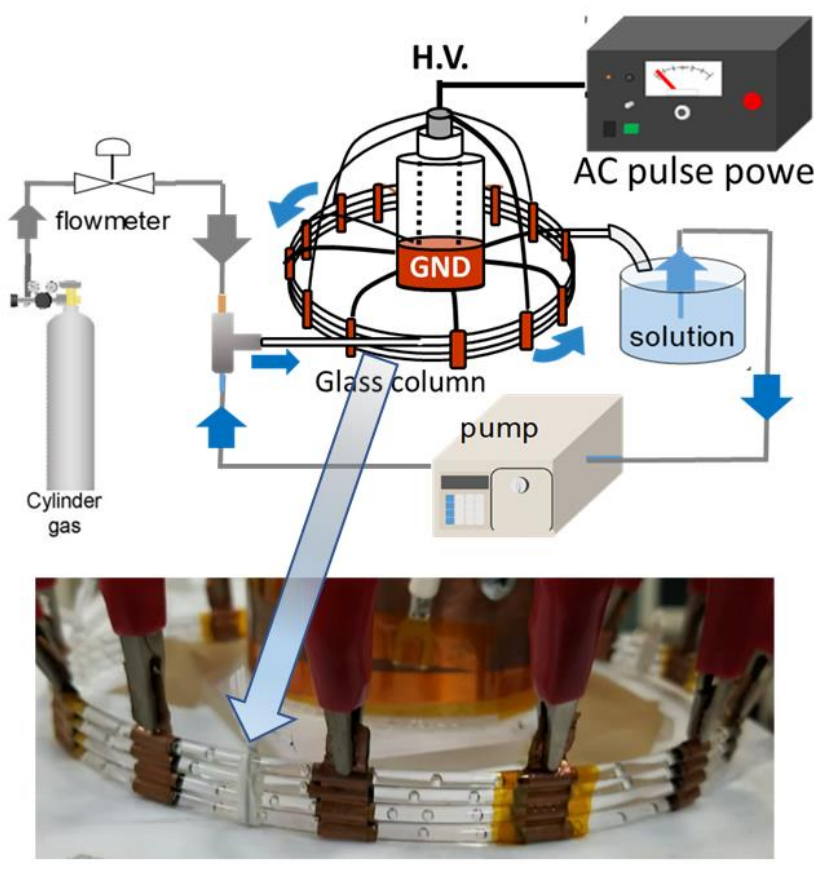

Fig. 1. Schematic diagram illustrating (a) straight and (b) helical types of plasma reactor [11-14].

Next, to generate discharge plasma in the gas-liquid slug-flow system, the bipolar high-voltage from pulsed power supply was introduced through a cooper sheet electrode to the slug flow reactor system. This electrode was placed on the outside of the glass capillary tube surface. The digital oscilloscope (TDS2024C, Tektronix Inc.) was employed to monitor the applied voltage and current during experiments. HR4000 Ocean Optics and
ICCD camera PI-MAX 512 devices also were used to capture the spectral analysis and the optical emission intensity distribution of discharge plasma in the slug flow reactor system, respectively. Although all experiments were carried out at room temperature, the temperatures of the starting materials and the solution products were monitored by using K-type thermocouple which assembled to the digital temperature controller (model TR-KN, AS-ONE Corp., Japan). The temperature (before and after treatment by a high voltage discharge plasma) was kept at around $25 \pm 2{ }^{\circ} \mathrm{C}$. The UV-vis spectrophotometer V-550 (Jasco Corporation, Japan) and the transmission electron microscopy (TEM) (JEM2100Plus, Japan Electronic Co., Ltd.) with energydispersive $\mathrm{X}$-ray spectroscopy devices were employed to analyze the aqueous solution products and to characterize the collected nanoparticle products.

\section{Reactive Oxidation Species}

Figure 2 displays the emission spectra of discharge plasma with diverse gaseous phases in the wavelength ranges from 200 to $900 \mathrm{~nm}$ that were captured from the gas-liquid bubble surface in the slug-flow reactor system. There are many ways to characterize the discharge plasma that was introduced on the surface of gas-liquid solution, and these characterization ways may give an effective information for the object which was contacted with a high voltage discharge plasma in the solution [23, 24]. Probably, the simplest technique to identify the reactive species generated from the interaction between the high voltage discharge plasma and gas-liquid water media is optical emission spectroscopy (OES) via optical fiber and spectrometer devices. As informed above, the reactive molecules and the reactive species with high reactivity were generated when the aqueous solution medium was treated by the high voltage discharge plasma in or over its surface. These reactive species may interact and react with the substances, especially organic compounds, dissolved in water medium.

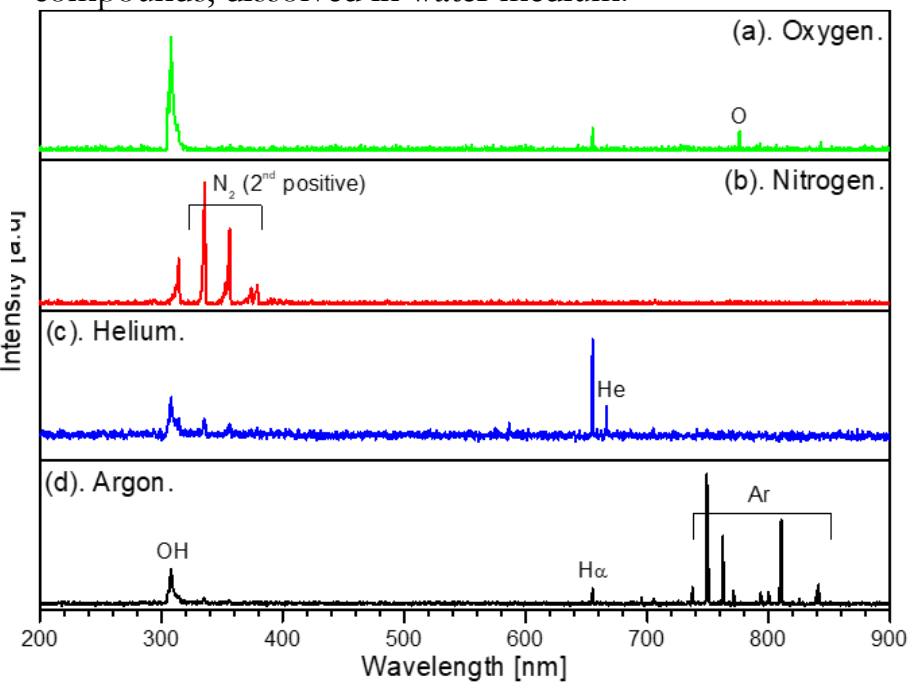

Fig. 2. Emission spectrum of pulsed discharge plasma on the water surface with various gases [11]. 
It can be seen in Fig. 2, the reactive molecules and the reactive species were discovered clearly at each OES spectrum. It means that the reactive radicals were successfully generated in the slug-flow reactor system by the applying high voltage discharge plasma onto the surface of gas-liquid water bubbles. When the argon gas was employed as a gas phase, the depopulation of $4 p$ level via multiple $4 \mathrm{p}-4 \mathrm{~s}$ transition processes occur. As a result, as shown in Fig. 2(d), the atomic argon emission was found dominantly in the infrared zone from 700 to $900 \mathrm{~nm}[25,26]$. At this condition, the dissociative excitation process of oxygen atom may also occurs resulting in the high-lying excited energy levels of atomic oxygen. Although, this dissociative excitation process was not occurred intensively, the spectral emission of oxygen element still can be observed by the OES spectrometer device and was shown in the infrared zones at around 845 and $777 \mathrm{~nm}$. Besides the spectral emission of oxygen element, the spectral emission in the visible zones at around $656 \mathrm{~nm}$ and $309 \mathrm{~nm}$ were also observed clearly in Fig. 2(d). These spectral emissions were associated with the existence of atomic hydrogen and hydroxyl radical species, respectively, in the slugflow reactor system. When the oxygen or helium gas was introduced in the slug-flow reactor system to generate bubble motions, the peaks spectral emission at the wavelengths of 777 and $667 \mathrm{~nm}$ that were corresponded to the atomic oxygen and atomic helium, respectively, can be observed clearly. At these OES spectra, the peak spectral emission at $309 \mathrm{~nm}$ was also found clearly. This peak revealed that the hydroxyl radical species was also generated when the oxygen or helium gas was employed as a gas phase in the slug-flow reactor system [25, 27]. As shown in Fig. 2(b), the spectral emission at the wavelength zones from 300 to $400 \mathrm{~nm}$ was observed prominently when the nitrogen gas was introduced in the slug-flow reactor system as a gas phase. It revealed that the atomic nitrogen species were generated abundantly. Hsieh el al. [26] conducted experiment to analyze the characteristic of discharge plasma in a gas-liquid water medium by using OES spectrophotometer in flow reactor system. They informed that the hydroxyl radical species can be formed from the transition of hydroxyl $\left(\mathrm{A}_{2} \Sigma^{+} \rightarrow \mathrm{X}_{2} \Pi\right)$ when the nitrogen gas was applied as a gas phase. Hence, as shown in this figure, although the strong emission at the wavelength from 300 to $400 \mathrm{~nm}$ corresponding to atomic nitrogen species was observed prominently, the spectral emission attributing to the hydroxyl radical species at the wavelength of $309 \mathrm{~nm}$ was still found clearly. Based on these OES spectra, it could be said that the variety of reactive species generation and the electronic transition phenomenon occur during the applying high voltage discharge plasma onto the liquid water solution [26].

Figure 2 also illustrated that the hydroxyl radicals seem to have the robust peak spectral emission in each OES spectra. It might be caused by the high conductivity of liquid solution as a medium, where the liquid environment with a high conductivity may act as a grounded electrode and may give a significant influence on the high voltage discharge plasma characteristics [26, $28,29]$. Lukes et al. [28] observed the activity of plasmachemical by applying pulsed discharge plasma in the liquid water as a medium. They informed that the increasing solution conductivity, which was attributed to the higher concentration of ions in the liquid water medium, can affect strongly the streamer propagation channel of discharge plasma. The shorter of streamer propagation channel might occur at the higher liquid water conductivity leads to the generation of the discharge plasma in a denser condition. As a result, the hydroxyl radical species can be generated abundantly at the higher liquid water conductivity. Jiang et al. [29] also informed that the generation of hydroxyl radical species was strongly influenced by the liquid water conductivity as a medium when they observed the remediation of wastewater by using the high voltage discharge plasma technology. They reported that the maximum value of hydroxyl radical species in the OES spectra was found when the wastewater remediation process was performed at the liquid water medium with the higher conductivity.
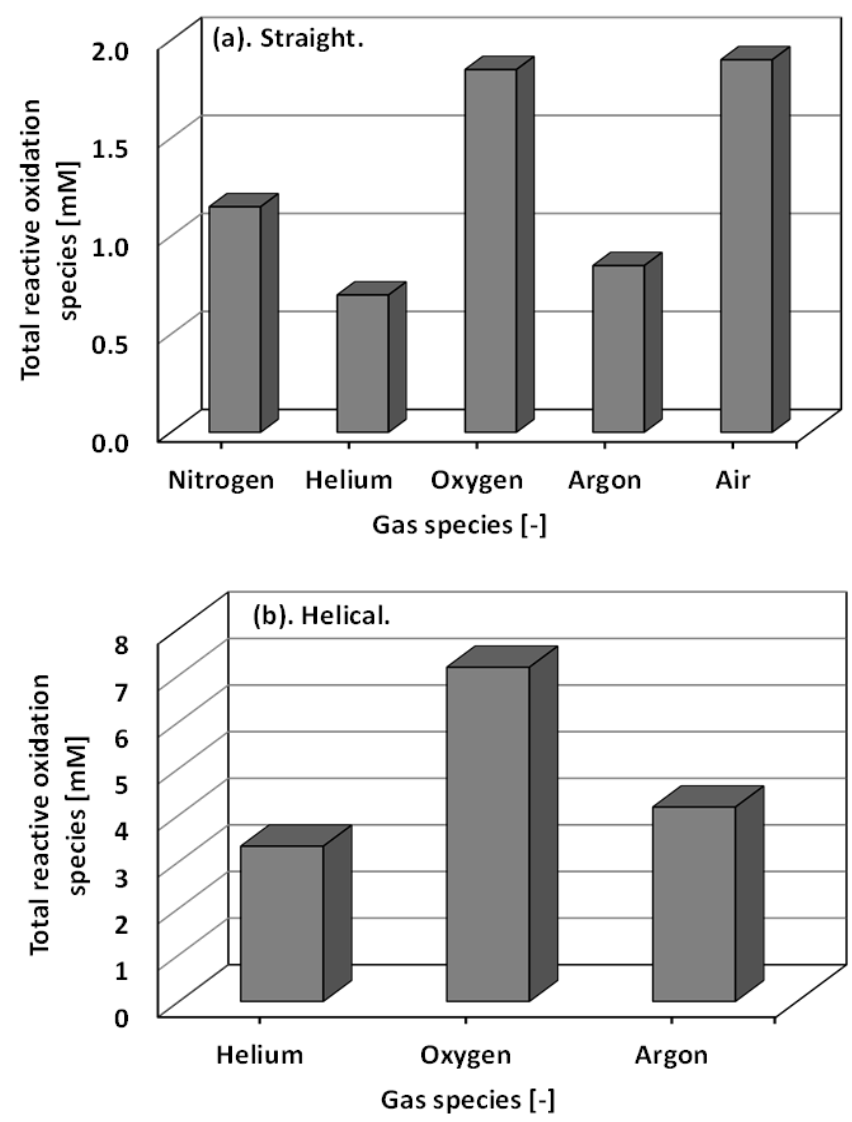

Fig. 3. Total amounts of reactive oxidation species [11, 12].

To analyze the total amounts of reactive oxidation species in the plasma apparatus, as a substitute for liquid water medium, the aqueous solution containing starch and potassium iodide was employed as a liquid phase in the slug-flow reactor system. When the high voltage discharge plasma was applied onto this aqueous solution, 
the color of this aqueous solution shifted into purple color. The shift in the color solution from transparent into purple color indicated that the aqueous solution interacts and reacts with reactive species that were formed during the applying high voltage discharge plasma in the slug-flow reactor system. Next, the total amounts of reactive oxidation species were determined based on the change of solution concentration (starch and potassium iodide) due to its reaction with the reactive oxygen species in the aqueous solution media [30, 31]. In brief, the simple reaction pathway can be described as follows. First, the oxidation reaction of iodine ion $\left(\mathrm{I}^{-}\right)$into the iodine molecule $\left(\mathrm{I}_{2}\right)$ by the generated reactive oxygen species occurs. The next reaction step is the formation of tri-iodide ion $\left(\mathrm{I}_{3}{ }^{-}\right)$from the interaction between the iodine ion excess and the iodine molecule. In the further reaction, the tri-iodide ion will react with starch molecule that was pointed out by the transformation of color solution from transparent into purple color.

Figure 3 displays the generated reactive oxidation species after the applying high voltage discharge plasma in the slug-flow reactor system. Since the different properties of each gas, the different gas type as a gas phase led to result in the different amount of the generated reactive oxidation species [32-34]. Takamatsu et al. [32] investigated the reactive oxidation species generated by a high voltage discharge plasma under various types of gas species. They reported that the type of gas environment strongly affects on the quantity and the variety of the reactive oxidation species during the applying high voltage discharge plasma on the aqueous solution surface. They explained that the formation of reactive oxidation species was started in the gas phase zone at the beginning of the applying high voltage discharge plasma on the aqueous solution surface. By the extension of this process, they were then transferred and distributed into the liquid phase zone. At the same time, the fresh reactive species was also formed. Depending on the properties of fresh reactive species, in the further process, the new reactive species will interact and react with each other to construct other chemical reactive species within a certain time. In most cases, the reactive species contains atomic oxygen, i.e. hydroxyl radical, metastable oxygen molecule, and atomic oxygen, are produced and detected prominently as reactive oxidation species products. As illustrated in Fig. 2, the OES spectrophotometer device detected that the hydroxyl radicals seemed to be the main reactive oxidation species during the high voltage discharge plasma treatment in the slug-flow reactor system at each experimental condition. Certainly, this process can give a beneficial result due to the hydroxyl radical can be acted as an important key in the water pollutant compound decomposition and the synthesis of nanoparticles via chemical reaction process with liquid water as a medium. Obviously, the generation of reactive oxidation species is higher when the oxygen or air gas was employed as a gas phase than the nitrogen, helium or argon gas was used as a gas phase in a straight type of slug-flow reactor system (see Fig. 3(a)). Here, as a substitute for natural air, the mock air was employed as a gas phase in this experiment. It was formed from the mixture of oxygen and nitrogen gases with a ratio of $20 \%$ and $80 \%$ for oxygen and nitrogen, respectively, due to the natural air normally consisted of oxygen and nitrogen as the main elements. When the high voltage discharge plasma was applied in the slug-flow reactor system with the oxygen gas as a gas phase, the oxygen molecule may undergo dissociation reaction to contribute for the generation of reactive oxidation species which has a long-lived period $[29,35,36]$. The similar reaction pathway may also occur when the mock air was acted as a gas phase due to the existence of oxygen element. Conversely, although the nitrogen, helium or argon gas might be also involved in the formation of reactive oxidation species, the generated reactive oxidation species usually has relatively short-lived period $[23,32$, 33]. This may be the main reason why the formation of reactive oxidation species is high when the oxygen gas or air was employed as a gas phase in this slug-flow reactor system.

As shown in Fig. 3, with the same type of gas, the concentrations of the generated reactive oxidation species increased significantly when the slug-flow reactor system with helical form was used to substitute the straight type of reactor during the applying high voltage discharge plasma. The concentrations of the generated reactive oxidation species in the straight type of reactor were $0.70,1.85$, and $0.85 \mathrm{mM}$ when the helium, oxygen, and argon were used as a gas phase, respectively. These concentrations increase drastically to $3.33,7.17$, and 4.17 $\mathrm{mM}$ with the similar type of gas phase when the high voltage discharge plasma was applied in the helical type of reactor at the same operating conditions. Note, the mock air or the nitrogen gas was not used as a gas phase in the discharge plasma experiments with the helical type of reactor. Besides the substitution of reactor type from the straight type with $25 \mathrm{~mm}$ length to the helical type with $2.0 \mathrm{~m}$ length can improve the contact area between gas-liquid water and applied discharge plasma, it seems that this substitution also can provide the longer residence time of bubble motion in the slug-flow reactor system from $3 \mathrm{~s}$ to $3 \mathrm{~min}$. This aspect is one of important factors in terms of applying high voltage discharge plasma to provoke the discharge plasma reactivity in gasliquid water system [37-39].

\section{Water Pollutants Decomposition}

Figure 4 displays the conversions of $\mathrm{CBB}$ and $\mathrm{MB}$ dye compounds after treatment by high voltage discharge plasma with various gas phases in the straight and helical types of slug-flow reactor system. As informed before, besides CBB was known as a prominent synthetic dye and widely applied in various industrial applications, this dye compound is recognized to be an organic pollutant in wastewater when this organic pollutant released into the water environment directly without some treatment. 
In addition to the highly toxic property to aquatic life, although at a low concentration, CBB dye compound may obstruct the penetration of light in the liquid water resulting in the decreasing photosynthesis activity $[17,40$, 41]. Consequently, the degradation process, it means decoloration process, for the dye compounds removal from wastewater stream including CBB dye compound is still to possess a great attention.

In the straight type of slug-flow reactor system (see Fig. 4(a)), it shows obviously that the different gas species as a gas phase resulted in the different CBB dye compound degradation rate. The degradation rate could approach to $77.62,29.31,78.00,28.00$, and $38.21 \%$ when the mock air, argon, oxygen, helium, or nitrogen gas, respectively, was introduced as a gas phase. These results indicated that the diffusion of generated reactive oxidation species in the gas phase occurs during the applying high voltage discharge plasma. It was then followed by the entering and dissolving the generated reactive oxidation species into liquid water solution through its surface layer. In the next step, these reactive oxidation species will interact and react with $\mathrm{CBB}$ dye compound in the liquid water as a medium lead to the CBB dye decoloration process [32, 42].
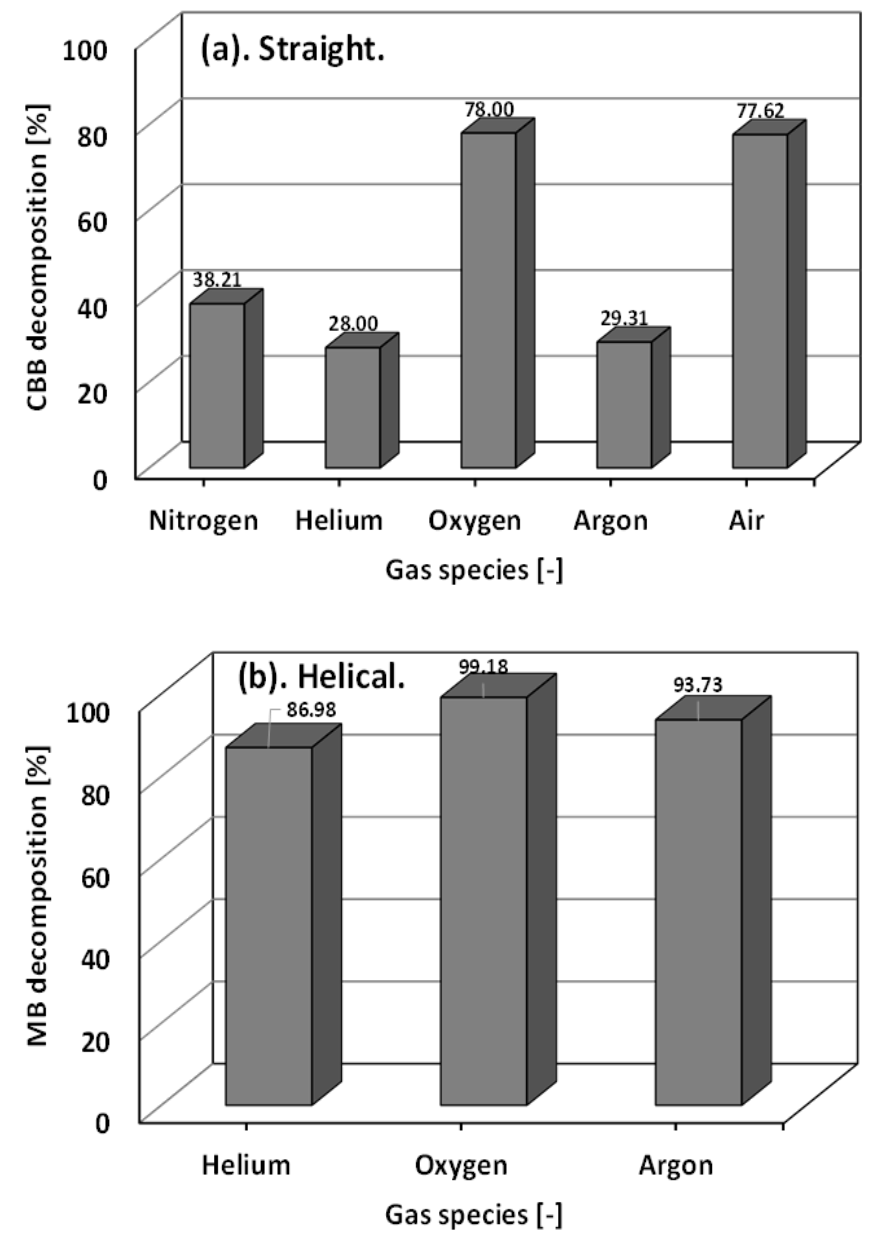

Fig. 4. Decomposition of $\mathrm{CBB}$ and $\mathrm{MB}$ dyes as water pollutant model compounds $[11,12]$.

From Fig. 4(a), it can be seen that the oxygen gas or mock air appears to give the higher rate of $\mathrm{CBB}$ dye compound degradation compared to other gas species when this gas was acted as a gas phase. It was well known that the oxygen base reactive oxidation species, i.e. hydrogen peroxide and ozone, possessed the high reactivity properties. These reactive species may interact and react intensively with organic compounds including $\mathrm{CBB}$ dye with liquid water as a medium. Probably, the oxygen base reactive oxidation species were produced abundantly during the applying high voltage discharge plasma in the slug-flow reactor system with an oxygen gas as a gas phase. As a result, the decoloration process of $\mathrm{CBB}$ dye compound can be proceeded effectively. In addition to this reaction, the reaction between the $\mathrm{CBB}$ dye compound via its functional group with the oxygen radicals can also occur directly that can enhance the decoloration process $[43,44]$. Since the mock air had an oxygen gas as a main component, the same reaction step process also might be occurred when the mock air was employed as a gas phase in the same reactor system. Hence, the decoloration of $\mathrm{CBB}$ dye compound was also obtained in high-rate degradation when the mock air was acted as a gas phase at the similar reactor system.

At the same operating parameters, the degradation rate of dye compound can increase significantly when the high voltage discharge plasma was operated in the slugflow reactor system with helical type. Note, in this reactor type, the solution of $\mathrm{MB}$ dye compound was applied as a starting material; and only the helium, argon, or oxygen gas was applied as a gas phase. Like the CBB dye compound, besides the toxic property to aquatic life, this dye compound also can decline the photosynthesis activity due to it able to obstruct the light penetration in the liquid water medium $[11,16]$. As shown in Fig. 4(b), the MB dye compound could be degraded over than $85 \%$ with the various gaseous as a gas phase. The degradation rate could approach to $86.98,93.73$, and $99.18 \%$ with helium, argon, or oxygen gas as a gas phase, respectively. This result confirms that the contact area and the contact time between gas-liquid water and applied discharge plasma in the high voltage discharge plasma technique are important factors for the decoloration of dye compounds. Based on the experimental results, it could be said that this decoloration technique with the straight or helical type reactor is a simpler and more effective to decompose the dye compounds compared to biodegradation, physical or chemical way [40, 45, 46]. Moon et al. [45] reported that the CBB dye compound decomposition could approach to $88 \%$ by using photocatalytic decomposition technique. However, this photocatalytic reaction needs long reaction time, around 200 min, with $\mathrm{pH}$ environment at 10. When the $\mathrm{pH}$ environment shifted to 2 , this technique can only decompose $\mathrm{CBB}$ dye compound at around $20 \%$ with the same reaction time. In biological process, Paz et al. [40] reported that, in addition to the complex process, the maximum removal of $\mathrm{CBB}$ dye compound at around $54 \%$ can be obtained after $72 \mathrm{~h}$. Furthermore, it seems that the application of high voltage discharge plasma in the slug-flow type reactor system is more effective than in 
the batch type reactor system for the water pollutant compound decomposition. Abdel-Fattah [47] employed the plasma jet at an atmospheric condition with helium gas to decompose $\mathrm{MB}$ dye compound in a batch reactor system. As starting solution, he used $30 \mathrm{ml}$ of MB dye compound solution with $35 \mathrm{ppm}$ concentration. When the flow rate of helium gas is $10 \mathrm{l} / \mathrm{min}$, the $\mathrm{MB}$ dye compound can be decomposed to $98.5 \%$ after $40 \mathrm{~min}$ treatment time. However, when the flow rate of helium gas was decreased to $2.5 \mathrm{l} / \mathrm{min}$, the degradation of $\mathrm{MB}$ dye compound was $60 \%$ with the same treatment time. Nishiyama et al. [48] also conducted experiment for the MB dye compound decomposition by using pulsed discharge plasma in the batch type reactor system. The argon gas at $4 \mathrm{l} / \mathrm{min}$ flow rate was introduced into the reactor to generate the bubbles. $1 \mathrm{ppm} \mathrm{MB}$ dye compound solution at $550 \mathrm{ml}$ volume was used as a feed solution. They reported that the MB dye compound can be converted to $90 \%$ after $30 \mathrm{~min}$ applying pulsed discharge plasma.

\section{Nanoparticle Synthesis}

As mentioned in the Introduction-Section, the high voltage discharge plasma in a slug-flow reactor system was also used to synthesize silver or gold nanoparticles. There are many techniques to produce metal nanoparticles including silver and gold, i.e. physical, biological, chemical techniques [49-51]. In physical technique, nanoparticles were usually formed by the reducing size of the bulk material. In this technique, since the chemical reagents or solvents were not involved during process, the contamination of the final products can be avoided. However, the physical technique is generally costly. In the biological or chemical technique, nanoparticles were formed by constructing atoms or molecules. The chemical technique is the most common technique to generate nanoparticles due to this technique is easy and convenience process and needs simple device. Nevertheless, in this technique, the chemical reagents or solvents were involved during nanoparticles generation. Hence, the hazardous residues might be generated and obtained at the end process. Compared to the physical or chemical technique, the biological technique was recognized as a cheap and an environmentally friendly technique. In the biological technique, the living organisms were employed to generate molecules to substitute the chemical reagents that were involved in the nanoparticle generation process. Nevertheless, in addition to the reproducibility process of this technique was limited, the biological technique may also result in the biological contaminant residues as undesired products. To overcome the problem, here, the generation silver or gold nanoparticles by the applying high voltage discharge plasma in a slug-flow reactor system with argon gas as a gas phase was demonstrated.

Figure 5 shows the UV-vis spectra of the collected solution products from slug-flow reactor system after treatment by high voltage discharge plasma. This analysis type was known as the simple and the first technique for metal nanoparticle characterization, including silver or gold, based on its physical phenomenon such as surface plasmon resonance. As shown in Fig. 5(a), it was clearly that the collected solution products seem to have the strong absorption light from 380 to $440 \mathrm{~nm}$ when the silver nitrate solution containing starch as a stabilizer was fed into the slug-flow reactor system and treated by high voltage discharge plasma. Conversely, the peak absorption light at the similar regions was found with the weak intensity when the silver nitrate solution without starch was fed as a starting material. This indicated that the silver nanoparticles were produced and dispersed in the collected solution products with the existence of starch in the starting material $[52,53]$. It seems that starch is able to adsorb and to bind the silver nanoparticles which were generated during the applying high voltage discharge plasma, where this adsorption process may avoid the growth and the agglomeration of these nanoparticle products.
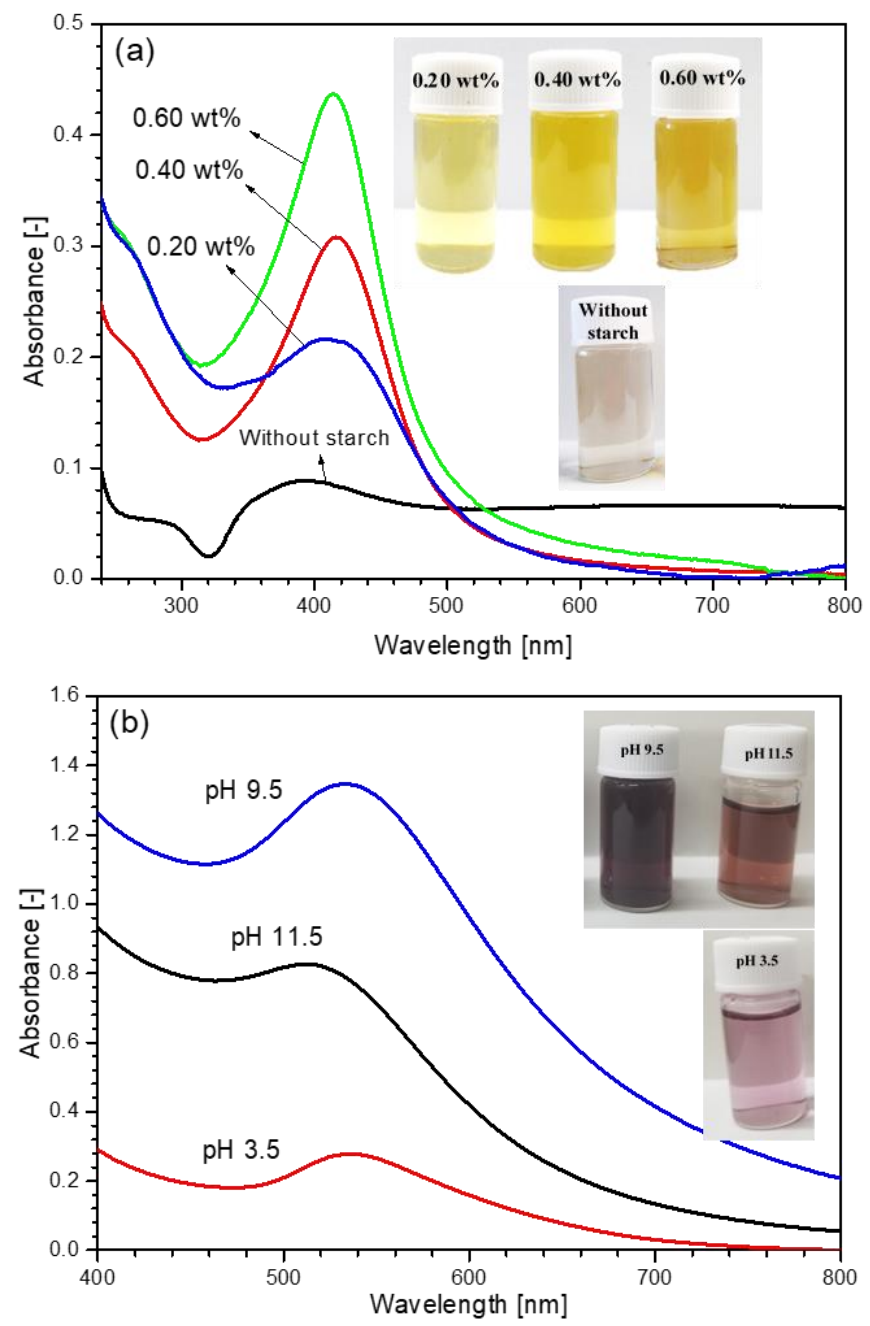

Fig. 5. UV-vis spectra of solution product containing (a) silver [13] and (b) gold nanoparticles [14].

Contrary to this, without the starch addition in the starting material, the silver nanoparticles underwent aggregation process via the formation of silver nanoparticle cluster [54-56]. Besides the UV-vis analysis, 
the energy-dispersive X-ray spectroscopy analysis (EDX) was also performed to characterize the collected particle products. This analysis technique is simple and able to identify the chemical composition of the various nanoparticles. Figure 6 displays the EDX spectrum of the generated silver nanoparticles produced from the starting material with the starch addition of $0.40 \mathrm{wt}^{\%} \%$. This figure exhibits that the strong signal at $3 \mathrm{keV}$ attributing to the silver element zone was clearly observed indicating the existence of silver particle in the in the collected solution product $[57,58]$. Other elements, i.e. carbon, oxygen, and copper elements, were also clearly observed in this spectrum. The oxygen and carbon elements might be originated from the starch molecule degradation during applying high voltage discharge plasma that may adhere to the surface of silver nanoparticle. While the peak spectrum attributing to copper element might be provenance from the TEM copper grid that was employed as a medium for the characterization of samples.

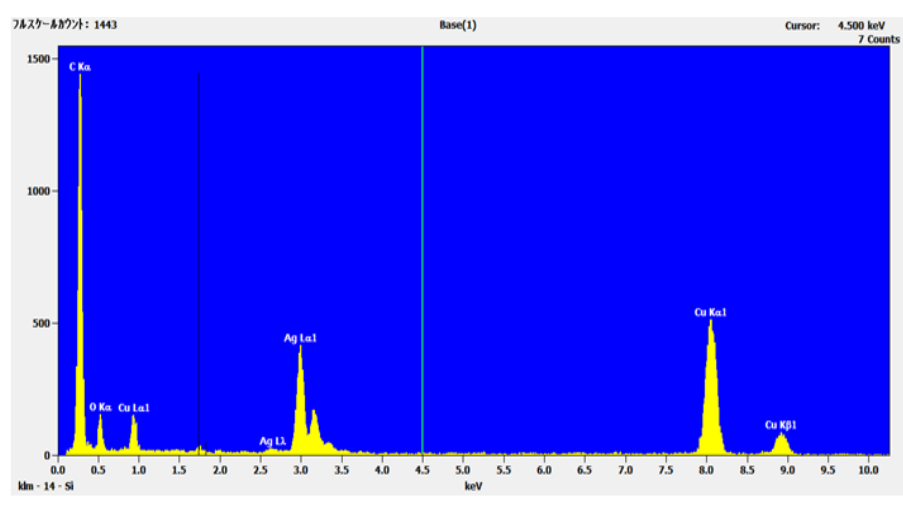

Fig. 6. EDX analysis of silver nanoparticle [13].

In addition to the UV-vis analysis result, by using a common digital camera (Olympus TG-3), it was clearly that the different color was found between the collected solution product from the starting material without and with the starch addition (see Fig. 5(a)). The photographs show that although the collected solution product from the starting material without the starch addition seems to possess a whitish or transparent gray color dominantly, in fact, the yellow color at the low intensity was still obtained in this solution product. In agreement with the UV-vis analysis result, it reveals that the silver nanoparticle was successfully produced and distributed in the collected solution product although in the low concentration. At the same operating conditions, the collected solution product with yellow color was obtained obviously when the starting material with the starch addition was flowed in the slug-flow reactor system. This result pointed out that the existence of starch in the starting material may lead to bind and to stabilize the generated silver nanoparticle resulting in the colloidal solution easily. Apparently, the collected solution product colors shifted from a clear light yellow to a muted yellow color with increasing the concentration of starch as a stabilizer. Since the colloidal solution color was generally associated with the surface plasmon resonance characteristic, the color shift of these solutions might be influenced by the generated silver nanoparticle amount in the solution. It seems that the large amount of the generated silver nanoparticle can be pointed out by the muted yellow color of the solution product due to the caping or the binding process of these nanoparticles might be occurred effectively at the higher starch concentration [56]. There was no precipitation during experiments, even after it was stored in several weeks, the sedimentation was also not found at each collected sample. Consequently, when the UV-vis spectrophotometer was used to analyze these collected solution products, the different peak intensities were obtained at the regions of 380 to $440 \mathrm{~nm}$ which were attributed to the presence of silver nanoparticle. At the highest starch addition concentration (0.60 $\mathrm{wt} \%)$, the sharp peak with a high intensity was observed clearly. Although the decreasing starch addition concentration was followed by the decreasing peak intensity at these regions (380 to $440 \mathrm{~nm}$ ), it seems that each collected solution product had the similar maximum wavelength at $418 \mathrm{~nm}$. Hence, it could be said that the starch addition at a certain amount might offer medium for the silver nanoparticle nucleation and bind them to avoid the agglomeration process and any further growth.

Like the collected solution product containing silver nanoparticles, the collected solution product obtained from the hydrogen tetrachloroaurate (III) tetrahydrate solution as a starting material was also analyzed by using $\mathrm{UV}$-vis spectroscopy equipment via the quartz cuvette device. Figure 5(b) shows the UV-vis spectra of the collected solution product containing gold nanoparticle at various $\mathrm{pH}$ values after the applying high voltage discharge plasma. It can be seen that the peak spectra at the wavelength regions between 510 and $550 \mathrm{~nm}$ were found at each collected sample. These peak spectral were associated with the presence of gold particle in the collected solution products where their optical absorption intensities were strongly affected by the dispersity, shape, and number of the gold particles [5961]. This figure also displays the collected solution product containing gold nanoparticle possessed the purple color owing to the surface plasmon resonance characteristic of gold particles [59, 62]. The purple color also indicates that the gold nanoparticles were dispersed in the collected solution product in stable form. As shown in Fig. 5(b), though the purple color could be observed in all solution products with the different $\mathrm{pH}$ conditions, obviously, the strongest purple color was appeared in the collected solution product with $\mathrm{pH}$ value of 9.5. It shows that the collected solution product also had the highest optical absorption intensity at this $\mathrm{pH}$ value. This result indicated that the gold nanoparticle organizational or structural might be affected by the $\mathrm{pH}$ value of solution which was used as a medium. Between the $\mathrm{pH}$ values of 3.5 (acid environment) and 11.5 (base environment), the collected solution product seems to possess the different color in slightly. At an acid environment, the collected solution product had purple 
color, while the red-purple color of the collected solution product was obtained in a base environment. They also had the distinct peak spectral intensity; in acid environment, the strong peak spectral intensity occurs at the wavelength of $535 \mathrm{~nm}$, and in base environment, the strong peak spectral intensity was found at the wavelength of $511 \mathrm{~nm}$. Since the increasing size of gold nanoparticles may promote the red shift of peak spectral absorption [59], it can be concluded that this experiment may result in the gold nanoparticle with the larger size in acid environments compared to those in base environments. Probably, the different reaction mechanism for the formation of gold nanoparticle by using this technique occurs [63-65]. At an acid environment, the gold nanoparticle was generated via the reduction reaction of tetrachloroaurate ion $\left(\mathrm{AuCl}_{4}{ }^{-}\right)$, while at a base environment, the gold nanoparticle was formed from the reduction reaction of gold ion complex $\left(\mathrm{Au}(\mathrm{OH})_{4}^{-}\right)$. These reaction mechanisms were expressed as follows [63-65]:

$$
\begin{aligned}
& \mathrm{AuCl}_{4}^{-}+3 \mathrm{H}^{\bullet}==>3 \mathrm{HCl}+\mathrm{Au}^{0}+\mathrm{Cl}^{-} \\
& \mathrm{Au}(\mathrm{OH})_{4}^{-}+3 \mathrm{H}^{\bullet}==>3 \mathrm{H}_{2} \mathrm{O}+\mathrm{Au}^{0}+\mathrm{OH}^{-}
\end{aligned}
$$

where reactions 9 and 10 take place in the acid environment (in this case of $\mathrm{pH}$ 3.5) and the base environment (in this case of $\mathrm{pH} 9.5$ or 11.5), respectively.

To understand the physical properties of silver or gold nanoparticles, i.e. size, shape, or dispersity, a few drops of the collected solution products were dropped on the TEM copper grid and then immediately dried in a vacuum desiccator at room temperature around overnight. Next, it was submitted into TEM equipment to observe their physical properties. In most cases, the TEM analysis was used to corroborate for the formation particle at the nanoscale. Even, this analysis may also offer the preliminary information for the size distribution of particles including silver and gold particles. Figures 7 and 8 exhibit the TEM images of silver and gold nanoparticle products with their size distributions at various operating conditions. It shows that the generated silver nanoparticles seem to have spherical morphology and they are well dispersed in the collected solution products at each operating condition. As mentioned above, the addition of starch in the starting material was acted as a stabilizer during the silver nanoparticles formation. Hence, the well dispersion of them in the collected solution products could be associated with the effective passivation by starch molecule during experimental process. The silver nanoparticle passivation process is able to suppress the aggregation and further growth of nascent silver particle. This passivation process occurs on its surface, where among the silver nanoparticle and the starch molecule interacts strongly through its active functional groups $[56,66,67]$. Nevertheless, as shown in Fig. 7, the agglomeration might be still occurred on the smaller particles resulting in the larger particles. This phenomenon occurs at each operating condition. As a result, in each population, the silver nanoparticles with the different sizes can be still found easily. Next, the ImageJ software was used to understand the size distribution of particle products based on the TEM images (Figure $7(\mathrm{a}-\mathrm{c})$ ). At least several hundred $(>300)$ of silver nanoparticles from each image in these figures were selected randomly. The distribution of sizes and the mean particle size of the generated silver nanoparticles obtained at each operating condition was exhibited in Fig. 7(d-f). It shows that the collected particle products had average diameter sizes of $8.00,6.04$, and $6.23 \mathrm{~nm}$ when the concentration of starch addition in the starting material was $0.6,0.4$, and $0.2 \mathrm{wt} \%$, respectively. It was observed that the different concentration of starch in the starting material did not give a high effect on the size of the silver nanoparticle products.

Hence, it could be said that whatever the starch addition amount in the starting material will be able to bind well the silver nanoparticle which were formed during the applying high voltage discharge plasma in the slug-flow reactor system. As a result, the absorption peak at the wavelength of $418 \mathrm{~nm}$ as an absorbance maximum for surface plasmon resonance of silver nanoparticles was not changed and still maintained (see Fig. 5(a)) [67]. Cheviron et al. [67] conducted experiments to observe the effect of carbohydrate addition on the silver nanoparticle synthesis in the aqueous solution at hot environments. When the size of silver nanoparticle products on average were less than 10 $\mathrm{nm}$, the carbohydrate addition amount in the starting material did not give a strong affect on the agglomeration during experimental process. Conversely, when the population size of silver nanoparticles on average were higher than $10 \mathrm{~nm}$, the amount of the presence of carbohydrate addition in the starting material may promote the agglomeration during experimental process.

As displayed in Fig. 8(a-c), the generated gold nanoparticle morphology is very similar with the morphology of silver nanoparticle products. Their morphologies are spherical shape in all operating conditions, and these nanoparticles were also obviously well dispersed in the collected solution products indicating that the gold nanoparticle was successfully produced through the self-assembly process with lysine compound as a capping agent [68-70]. Selvakannan et al. [68] did experiment to cap gold nanoparticle by using amino acid with lysine as a model compound in a liquid water as a medium. With lysine compound as a capping agent, the gold nanoparticles were successfully assembled in aqueous environment via the reduction reaction of gold ion by using sodium borohydride at around isoelectric point $(\mathrm{pI})$ of lysine compound. They mentioned the gold nanoparticles formation mechanism via self-assembly process. The mechanism was initiated by the binding process of gold nanoparticle via its surface by one amino $\left(-\mathrm{NH}_{2}\right)$ group as a reactive site in lysine compound. In the next process, the carboxyl $(-\mathrm{COOH})$ group and the terminal amino group interact 
with the reactive sites of lysine compound to form hydrogen bonds where this lysine compound was bound to the adjacent gold nanoparticles through their surface. In view of this reaction mechanism, the formation of lysine molecule layers at the adjacent gold nanoparticles might be occurred, and it was exhibited in the Fig. 9. It seems that the lysine molecule layer possessed 1.5 to 2.0 $\mathrm{nm}$ the interparticle space when the experiment was carried out at the environment with $9.5 \mathrm{pH}$ value $[71,72]$ This interparticle space was determined by using the Gatan microscopy suite software which was installed the TEM device.

Like the size determination of silver nanoparticles, the size of the generated gold nanoparticles was also determined by using ImageJ software based on their TEM images. At each operating condition, it showed clearly that each population of gold nanoparticles had the different size (see Fig. 8(d-f)). On average, the size of the generated gold nanoparticles was $9.83,5.41$, and $4.51 \mathrm{~nm}$ when the experiments were conducted at the $\mathrm{pH}$ values of $3.5,9.5$, and 11.5, respectively. This different size might be associated with the number of electric charges from the lysine compound which was affected by the $\mathrm{pH}$ value of medium. At the high $\mathrm{pH}$ environments, the lysine molecule had negatively charged, and conversely, the electric charged of lysine molecule shifted to positive charged at the low $\mathrm{pH}$ environments. Consequently, the balance of the number electric charge from lysine molecule was not reached at each environment resulting in the different size of gold nanoparticle products [68-70]. As shown in Figure 8, the largest diameter size of the generated gold nanoparticles was obtained at the low $\mathrm{pH}$ environment ( $\mathrm{pH}$ 3.5). It was well known that when the metal nanoparticles including gold were functionalized with amino acid, i.e. lysine, may promote self-assemble process via the formation of hydrogen bonds with the amine and/or carboxyl groups that can be found in the chemical structure of lysine [73, 74]. At the low $\mathrm{pH}$ environment, the carboxyl group did not undergo reaction dissociation; therefore, the interaction of gold nanoparticles and carboxyl group could be avoided. Nevertheless, the zwitterionic species is still able to activate the amine group to interact with the gold nanoparticles. As a result, the agglomeration of gold nanoparticles is still occurred to result in the bigger diameter size. On the contrary, the carboxyl group underwent reaction dissociation under base conditions, it means at the high $\mathrm{pH}$ environment ( $\mathrm{pH} 9.5$ or 11.5). This reaction can give a beneficial effect, where this reaction dissociation can obstruct the crosslinking reaction through the binding of the amine group and the gold nanoparticles [73, 74]. Next, the generated gold nanoparticles with the smaller diameter size were formed when the experiments were carried out at the high $\mathrm{pH}$ environment.

\section{Conclusions and Future Directions}

The high voltage discharge plasma in the slug-flow reactor system was successfully employed as a medium to decompose water pollutant compounds and to synthesize metal nanoparticles. The experiments were carried out at room temperature with the diverse gas types as gas phases. The reactive oxidation species, mainly the oxygen base reactive species, were obviously observed and identified by the OES device at each gas type, where the generation rate of them can be improved significantly by changing the reactor type from straight to helical form. The UV-vis spectra illustrated that the $\mathrm{CBB}$ or MB dye removal rate had order from oxygen $>$ air $>$ nitrogen $>$ argon $>$ helium. Furthermore, the silver or gold nanoparticles were also successfully formed by this plasma reactor system, except the gold nanoparticles produced at an acid environment, their diameter sizes were less than $10 \mathrm{~nm}$ on average. Although the high voltage discharge plasma was successfully applied in the slug-flow reactor system and employed as a medium to decompose water pollutant compounds and to synthesize metal nanoparticles, several challenges remain, particularly since most high voltage discharge plasma systems being constructed for small-scale processes, including our discharge plasma system. Therefore, more research efforts were needed to successfully generate plasma technology that is more appropriate for industrial-scale applications. However, it can be proposed that our high voltage discharge plasma system is usable technique for advanced organic compounds degradation and nanoparticles synthesis using the utilizing electric field generated by high voltage discharge plasma.

\section{Acknowledgement}

This work was supported by JSPS KAKENHI (Grant Number JP20H02515), JST SICORP (Grant Number JPMJSC18H1), Japan, and Sino - Japanese Joint Research Platform on energy and environmental industry, Grant/Award Number: 2017YFE012710. 

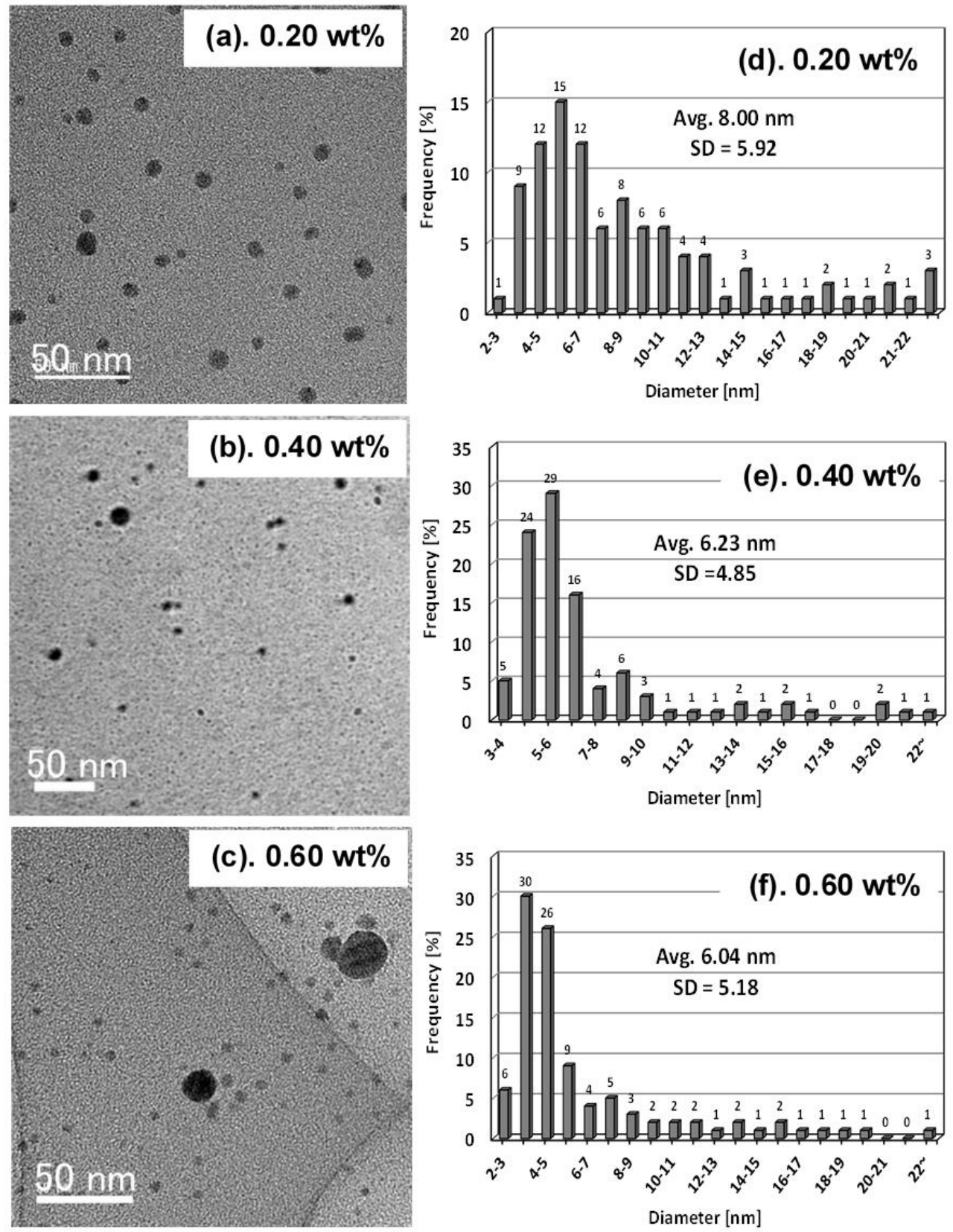

Fig. 7. TEM images and particle size distributions of silver nanoparticle products [13]. 

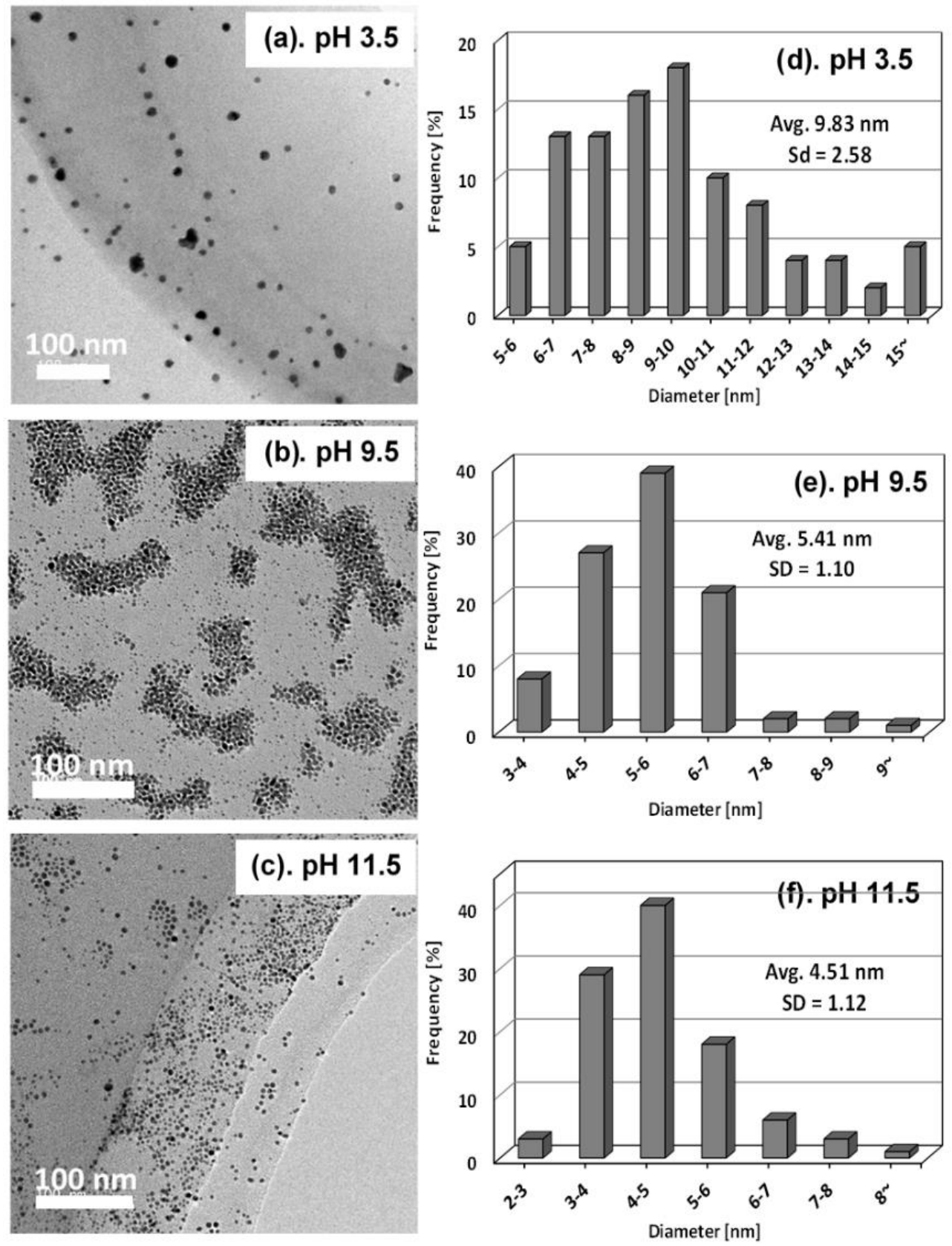

Fig. 8. TEM images and particle size distributions of gold nanoparticle products [14].

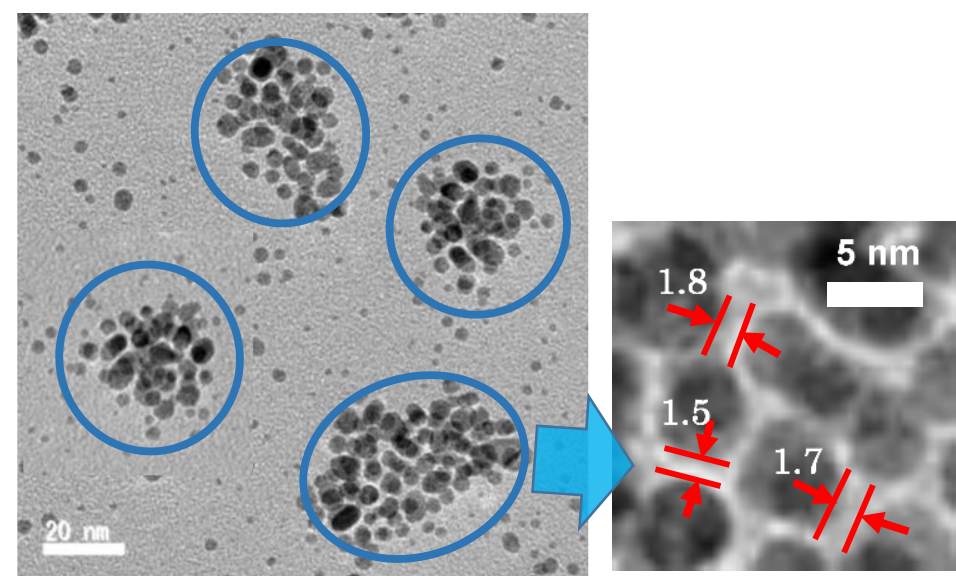

Fig. 9. Interparticle distance of gold nanoparticle products [14]. 


\section{References}

[1] B. R. Locke and S. M. Thagard, "Analysis and review of chemical reactions and transport processes in pulsed electrical discharge plasma formed directly in liquid water," Plasma Chemistry and Plasma Processing, vol. 32, no. 5, pp. 875-917, Aug. 2012.

[2] P. J. Bruggeman, M. J. Kushner, B. R. Locke, J. G. E. Gardeniers, W. G. Graham, D. B. Graves, R. C. H. M. Hofman-Caris, D. Maric, J. P. Reid, E. Ceriani, D. Fernandez Rivas, J. E. Foster, S. C. Garrick, Y. Gorbanev, S. Hamaguchi, F. Iza, H. Jablonowski, E. Klimova, J. Kolb, F. Krcma, P. Lukes, Z. Machala, I. Marinov, D. Mariotti, S. Mededovic Thagard, D. Minakata, E. C. Neyts, J. Pawlat, Z. Lj. Petrovic, R. Pflieger, S. Reuter, D. C. Schram, S. Schroter, M. Shiraiwa, B. Tarabova, P. A. Tsai, J. R. R. Verlet, T. von Woedtke, K. R. Wilson, K. Yasui, and G. Zvereva, "Plasma-liquid interactions: a review and roadmap," Plasma Sources Sci. Technol., vol. 25, no. 5, pp. 053002, Sept. 2016.

[3] C. San Wong and R. Mongkolnavin, Elements of Plasma Technology. Springer Science+Business Media Singapore Pte Ltd., 2016, pp. 2-6.

[4] Y. Hayashi, N. Takada, Wahyudiono, H. Kanda, and M. Goto, "Synthesis of hydrophilic carbon nanoparticles from amino acids by pulsed arc discharge over aqueous solution in argon under near-critical pressure," J. Supercrit. Fluids, vol. 120, no. 2, pp. 403-407, Feb. 2017.

[5] A. Claverie, J. Deroy, M. Boustie, G. Avrillaud, A. Chuvatin, E. Mazanchenko, G. Demol, and B. Dramane, "Experimental characterization of plasma formation and shockwave propagation induced by high power pulsed underwater electrical discharge," Rev. Sci. Instrum., vol. 85, p. 063701, June 2014.

[6] B. Sun, Y. Xin, X. Zhu, Z. Gao, Z. Yan, and T. Ohshima, "Effects of shock waves, ultraviolet light, and electric fields from pulsed discharges in water on inactivation of Escherichia coli," Bioelectrochem., vol. 120, pp. 112-119, Apr. 2018.

[7] J. Fan, H. Wu, R. Liu, L. Meng, and Y. Sun, "Review on the treatment of organic wastewater by discharge plasma combined with oxidants and catalysts," Environ. Sci. Pollut. Res., vol. 28, pp. 25222548, Jan. 2021.

[8] H. Nishiyama, R. Nagai, K. Niinuma, and H. Takana, "Characterization of DBD multiple bubble jets for methylene blue decolorization," Journal of Fluid Science and Technology, vol. 8, no. 1, pp. 65-74, Mar. 2013.

[9] B. R. Locke, "Environmental Applications of Electrical Discharge Plasma with Liquid Water," Int. J. Plasma Environ. Sci. Technol., vol.6, no.3, pp. 194203, Dec. 2012.

[10] R. J. Wandell, S. Bresch, H. Wang, V. Babicky, P. Lukes, and B. R. Locke, "The effects of pulse frequency on chemical species formation in a nanosecond pulsed plasma gas-liquid film reactor," Int. J. Plasma Environ. Sci. Technol., vol. 14, p. e01008, Apr. 2020.

[11] Wahyudiono, K. Mano, Y. Hayashi, M. Yamada, S. Takahashi, N. Takada, H. Kanda, and M. Goto, "Atmospheric-pressure pulsed discharge plasma in capillary slug flow system for dye decomposition," Chem. Eng. Process. Process Intensif., vol. 135, pp. 133140, Jan. 2019.

[12] M. Yamada, Wahyudiono, S. Machmudah, H. Kanda, and M. Goto, "Nonthermal atmospheric pressure plasma for methylene blue dye decolourisation using a slug flow reactor system," Plasma Chemistry and Plasma Processing, vol. 40, no.4, pp. 985-1000, Feb. 2020.

[13] M. Yamada, S. Takahashi, Wahyudiono, N. Takada, H. Kanda, and M. Goto, "Synthesis of silver nanoparticles by atmospheric-pressure pulsed discharge plasma in slug flow system," Jpn. J. Appl. Phys., vol. 58, no. 1, pp. 016001(1-8), Nov. 2019.

[14] M. Yamada, Wahyudiono, S. Machmudah, H. Kanda, Y. Zhao, and M. Goto, "Atmosphericpressure pulsed discharge plasma in a slug flow reactor system for the synthesis of gold nanoparticles," ACS Omega, vol. 5, no. 28, pp. 17679-17685, July 2020.

[15] M. A. Kamboh, W. A. Wan Ibrahim, H. Rashidi Nodeh, L. A. Zardari, S. T. H. Sherazi, and M. M. Sanagi, "p-Sulphonatocalix [8] arene functionalized silica resin for the enhanced removal of methylene blue from wastewater: equilibrium and kinetic study," Sep. Sci. Technol., vol. 54, no. 14, pp. 22402251, Jan. 2019.

[16] F. Afshariani and A. Roosta, "Experimental study and mathematical modeling of biosorption of methylene blue from aqueous solution in a packed bed of microalgae Scenedesmus," J. Cleaner Prod., vol. 225, pp. 133-142, July 2019.

[17] M. P. Rayaroth, U. K. Aravind, and C. T. Aravindakumar, "Sonochemical degradation of Coomassie Brilliant Blue: Effect of frequency, power density, $\mathrm{pH}$ and various additives," Chemosphere, vol. 119, pp. 848-855, Jan. 2015.

[18] J. S. Duhan, R. Kumar, N. Kumar, P. Kaur, K. Nehra, and S. Duhan, "Nanotechnology: The new perspective in precision agriculture," Biotechnol. Rep, vol. 15, pp. 11-23, Sept. 2017.

[19] Q. H. Tran, V. Q. Nguyen, and A. T. Le, "Silver nanoparticles: synthesis, properties, toxicology, applications and perspectives," Adv. Nat. Sci: Nanosci. Nanotechnol., vol. 4, no. 3, pp. 033001, May 2013.

[20] K. Kalimuthu, B. S. Cha, S. Kim, and K. S. Park, "Eco-friendly synthesis and biomedical applications of gold nanoparticles: A review," Microchem. J., vol. 152, pp. 104296, Jan. 2020.

[21] Y. Hayashi, N. Takada, Wahyudiono, H. Kanda and M. Goto, "Hydrogen Peroxide formation by electric 
discharge with fine bubbles," Plasma Chem. Plasma Process., vol. 37, no. 1, pp. 125-135, Jan. 2017.

[22] B. R. Locke, M. Sato, P. Sunka, M. R. Hoffmann, and J. S. Chang, "Electrohydraulic discharge and nonthermal plasma for water treatment," Ind. Eng. Chem. Res., vol. 45, no. 3, pp. 882-905, Jan. 2006.

[23] T. Kaneko, S. Sasaki, K. Takashima, and M. Kanzaki, "Gas-liquid interfacial plasmas producing reactive species for cell membrane permeabilization," J. Clin. Biochem. Nutr., vol. 60, no. 1, pp. 3-11, Jan. 2017.

[24] J. Liu, B. He, Q. Chen, J. Li, Q. Xiong, G. Yue, G.X. Zhang, S. Yang, H. Liu, and Q. H. Liu, "Direct synthesis of hydrogen peroxide from plasma-water interactions," Sci. Rep., vol. 6, pp. 38454, Dec. 2016.

[25] A. Sarani, A.Y. Nikiforov, and C. Leys, "Atmospheric pressure plasma jet in Ar and $\mathrm{Ar} / \mathrm{H}_{2} \mathrm{O}$ mixtures: Optical emission spectroscopy and temperature measurements," Phys. Plasmas, vol. 17, pp. 063504, June 2010.

[26] K. C. Hsieh, H. Wang, and B. R. Locke, "Analysis of electrical discharge plasma in a gas-liquid flow reactor using optical emission spectroscopy and the formation of hydrogen peroxide," Plasma Process. Polym., vol. 13, no. 9, pp. 908-917, Sept. 2016.

[27] V. Horvatic, S. Muller, D. Veza, C. Vadla, and J. Franzke, "Atmospheric helium capillary dielectric barrier discharge for soft ionization: Broadening of spectral lines, gas temperature and electron number density," J. Anal. At. Spectrom., vol. 29, no. 3, pp. 498-505, Jan. 2014.

[28] P. Lukes, M. Clupek, V. Babicky, I. Sisrova, and V. Janda, "The catalytic role of tungsten electrode material in the plasma chemical activity of a pulsed corona discharge in water," Plasma Sources Sci. Technol., vol. 20, pp. 034011, Apr. 2011.

[29] B. Jiang, J. Zheng, S. Qiu, M. Wu, Q. Zhang, Z. Yan, and Q. Xue, "Review on electrical discharge plasma technology for wastewater remediation," Chem. Eng. J., vol. 236, pp. 348-368, Jan. 2014.

[30] T. Kawasaki, W. Eto, M. Hamada, Y. Wakabayashi, Y. Abe, and K. Kihara, "Detection of reactive oxygen species supplied into the water bottom by atmospheric non-thermal plasma jet using iodine-starch reaction," Jpn. J. Appl. Phys, vol. 54, p. 086201, July 2015.

[31] Y. Asakura, T. Nishida, T. Matsuoka, and S. Koda, "Effects of ultrasonic frequency and liquid height on sonochemical efficiency of large-scale sonochemical reactors," Ultrason. Sonochem., vol. 15, no. 3, pp. 244-250, Mar. 2008.

[32] T. Takamatsu, K. Uehara, Y. Sasaki, H. Miyahara, Y. Matsumura, A. Iwasawa, N. Ito, T. Azuma, M. Kohno, and A. Okino, "Investigation of reactive species using various gas plasmas," RSC Adv., vol. 4, no. 75, pp. 39901-39905, Aug. 2014.

[33] V. V. Kovacevic, B. P. Dojcinovic, M. Jovic, G. M. Roglic, B. M. Obradovic, and M. M. Kuraica,
"Measurement of reactive species generated by dielectric barrier discharge in direct contact with water in different atmospheres." J. Phys. D: Appl. Phys., vol. 50, no. 15, p. 155205, Mar. 2017.

[34] S. Mohades, A. M. Lietz, and M. J. Kushner, "Generation of reactive species in water film dielectric barrier discharges sustained in argon, helium, air, oxygen and nitrogen," J. Phys. D: Appl. Phys., vol. 53, no. 43, p. 435206, Aug. 2020.

[35] Y. Cao, G. Qu, T. Li, N. Jiang, and T. Wang, "Review on reactive species in water treatment using electrical discharge plasma: Formation, measurement, mechanisms and mass transfer," Plasma Sci. Technol., vol. 20, no. 10, p. 103001, Aug. 2018.

[36] C. C. Lai, Y. X. Deng, and Y. H. Liao, "A study on the influence of gas mixtures on the property of plasma-activated water," Plasma Processes Polym., vol. 17, no. 2, pp. 1900196, Feb. 2020.

[37] H. Zhang, Y. Liu, X. Cheng, A. Zhang, X. Li, J. Liu, S. Cai, C. Yang, S. Ognier, and P. Li, "Degradation of phenol in water using a novel gas-liquid twophase dielectric barrier discharge plasma reactor," Water Air Soil Pollut., vol. 229, no. 314, pp. 1-12, Sept. 2018.

[38] F. Bilea, C. Bradu, N. B. Mandache, and M. Magureanu, "Characterization of the chemical activity of a pulsed corona discharge above water," Chemosphere, vol. 236, p. 124302, Dec. 2019.

[39] J. P. Liang, Z. L. Zhao, X. F. Zhou, D. Z. Yang, H. Yuan, W. C. Wang, and J. J. Qiao, "Comparison of gas phase discharge and gas-liquid discharge for water activation and methylene blue degradation," Vacuum, vol. 181, p. 109644, Nov. 2020.

[40] A. Paz, J. Carballo, M. J. Perez, and J. M. Dominguez, "Biological treatment of model dyes and textile wastewaters," Chemosphere, vol. 181, pp. 168-177, Aug. 2017.

[41] G. Saxena, R. Kishor, and R. N. Bharagava, Bioremediation of industrial waste for environmental safety. Volume I: Industrial $W$ aste and Its Management. Springer Nature Singapore Pte Ltd., 2020, pp. 326328.

[42] Wahyudiono, S. Machmudah, and M. Goto, "Pulsed discharge plasma over a water surface induces decoloration of dyes," J. Phys. Conf. Ser., vol. 441, p. 012008, Oct. 2013.

[43] D. B. Graves, "The emerging role of reactive oxygen and nitrogen species in redox biology and some implications for plasma applications to medicine and biology," J. Phys. D Appl. Phys., vol. 45, no. 26, p. 263001, June 2012.

[44] J. L. Brisset and E. Hnatiuc, "Peroxynitrite: A re-examination of the chemical properties of non-thermal discharges burning in air over aqueous solutions," Plasma Chem. Plasma Proc., vol. 32, pp. 655-674, May 2012.

[45] Y. E. Moon, G. Jung, J. Yun, and H. I. Kim, "Poly (vinyl alcohol)/poly (acrylic acid)/ $\mathrm{TiO}_{2} /$ graphene 
oxide nanocomposite hydrogels for $\mathrm{pH}$-sensitive photocatalytic degradation of organic pollutants," Mater. Sci. Eng.: B, vol. 178, no. 17, pp. 1097-1103, Oct. 2013.

[46] H. Tahir and U. Alam, "Lignocellulosic: Non-conventional low cost biosorbent for the elution of Coomassie Brilliant Blue (R-250)," Int. J. Chem., vol. 6, no. 2, pp. 56-72, Mar. 2014.

[47] E. Abdel-Fattah, "Atmospheric pressure helium plasma jet and its applications to methylene blue degradation," J. Electrostat., vol. 101, pp. 103360, Sept. 2019.

[48] H. Nishiyama, R. Nagai, and H. Takana, "Characterization of a multiple bubble jet with a streamer discharge," IEEE Trans. Plasma Sci., vol. 39, no. 11, pp. 2660-2661, Nov. 2011.

[49] Wahyudiono, H. Kondo, M. Yamada, N. Takada, S. Machmudah, H. Kanda, and M. Goto, "Dc-plasma over aqueous solution for synthesis of titanium dioxide nanoparticles under pressurized argon," ACS Omega, vol. 5, no. 10, pp. 5443-5451, Mar. 2020.

[50] S. Machmudah, Wahyudiono, N. Takada, H. Kanda, K. Sasaki, and M. Goto, "Fabrication of gold and silver nanoparticles with pulsed laser ablation under pressurized $\mathrm{CO}_{2}, "$ Adv. Nat. Sci: Nanosci. Nanotechnol., vol. 4, no. 4, p. 045011, Oct. 2013.

[51] M. Bilal and H. M. N. Iqbal, "Chemical, physical, and biological coordination: An interplay between materials and enzymes as potential platforms for immobilization," Coord. Chem. Rev., vol. 388, pp. 1-23, June 2019.

[52] L. D. Khemani, M. M. Srivastava, and S. Srivastava, Chemistry of Phytopotentials: Health, Energy and Environmental Perspectives. Springer-Verlag $\mathrm{GmbH}$ Berlin Heidelberg, Germany, 2012, pp. 155.

[53] Z. Jian, Z. Xiang, and W. Yongchang, "Electrochemical synthesis and fluorescence spectrum properties of silver nanospheres," Microelectron. Eng., vol. 77, no. 1, pp. 58-62, Jan. 2005.

[54] P. Mendis, R. M. de Silva, K. M. N. de Silva, L. A. Wijenayaka, K. Jayawardana, and M. Yan, "Nanosilver rainbow: a rapid and facile method to tune different colours of nanosilver through the controlled synthesis of stable spherical silver nanoparticles," RSC Adv., vol. 6, no. 54, pp. 48792 48799, May 2016.

[55] Y. L. Thong, O. H. Chin, B. H. Ong, and N. M. Huang, "Synthesis of silver nanoparticles prepared in aqueous solutions using helium dc microplasma jet," Jpn. J. Appl. Phys., vol. 55, no. 1S, p. 01AE19, Jan. 2016.

[56] V. S. S. K. Kondeti, U. Gangal, S. Yatom, and P. J. Bruggeman, "Ag+ reduction and silver nanoparticle synthesis at the plasma-liquid interface by an RF driven atmospheric pressure plasma jet: Mechanisms and the effect of surfactant," J. Vac. Sci. Technol., vol. 35, no. 6, p. 061302, July 2017.
[57] H. M. M. Ibrahim, "Green synthesis and characterization of silver nanoparticles using banana peel extract and their antimicrobial activity against representative microorganisms," J. Radiat. Res. Appl. Sci., vol. 8, no. 3, pp. 265-275, Jan. 2015.

[58] P. Magudapatty, P. Gangopadhyayrans, B. K. Panigrahi, K. G. M. Nair, and S. Dhara, "Electrical transport studies of $\mathrm{Ag}$ nanoclusters embedded in glass matrix," Physica, vol. 299, no. 1-2, pp. 142-146, May 2001.

[59] P. K. Jain, K. S. Lee, I. H. El-Sayed, and M. A. ElSayed, "Calculated absorption and scattering properties of gold nanoparticles of different size, shape, and composition: Applications in biological imaging and biomedicine," J. Phys. Chem. B., vol. 110, pp. 7238-7248, Mar. 2006.

[60] A. Wright, J. Gabaldon, D. B. Burckel, Y. B. Jiang, Z. R. Tian, J. Liu, C. Jeffrey Brinker, and H. Fan, "Hierarchically organized nanoparticle mesostructure arrays formed through hydrothermal self assembly," Chem. Mater., vol. 18, no. 13, pp. 3034-3038, June 2006.

[61] G. Ramirez-Garcia, M. A. Honorato-Colin, E. De la Rosa, T. Lopez-Luke, S. S. Panikar, J. de Jesus Ibarra-Sanchez, and V. Piazza, "Theranostic nanocomplex of gold-decorated upconversion nanoparticles for optical imaging and temperaturecontrolled photothermal therapy," J. Photochem. Photobiol. A Chem., vol. 384, p. 112053, Nov. 2019.

[62] S. Wang, H. Zhang, W. Li, Z. Birech, L. Ma, D. Li, S. Li, L. Wang, J. Shang, and J. Hu, "A multichannel localized surface plasmon resonance system for absorption metric determination of abscisic acid by using gold nanoparticles functionalized with a polyadenine-tailed aptamer," Microcbim. Acta, vol. 187, no. 20, Jan. 2020.

[63] M. A. Bratescu, S. P. Cho, O. Takai, and N. Saito, "Size-controlled gold nanoparticles synthesized in solution plasma," J. Phys. Chem. C, vol. 115, no. 50, pp. 24569-24576, Nov. 2011.

[64] K. Moore Tibbetts, B. Tangeysh, J. H. Odhner, and R. J. Levis, "Elucidating strong field photochemical reduction mechanisms of aqueous [AuCl4]?: Kinetics of multiphoton photolysis and radical mediated reduction," J. Phys. Chem. A, vol. 120, no. 20, pp. 3562-3569, May 2016.

[65] P. Xie, Y. Qi, R. Wang, J. Wu, and X. Li, “Aqueous Gold Nanoparticles Generated by AC and PulsePower-Driven Plasma Jet," Nanomaterials, vol. 9, no. 10, p. 1488, Oct. 2019.

[66] G. A. Valencia, L. C. de Oliveira Vercik, R. Ferrari, and A. Vercik, "Synthesis and characterization of silver nanoparticles using water-soluble starch and its antibacterial activity on Staphylococcus aureus," Starch, vol. 65, no. 11-12, pp. 931-937, Nov. 2013.

[67] P. Cheviron, F. Gouanve, and E. Espuche, "Green synthesis of colloid silver nanoparticles and resulting biodegradable starch/silver 
nanocomposites," Carbohydr. Polym., vol. 108, pp. 291-298, Aug. 2014.

[68] P. R. Selvakannan, S. Mandal, S. Phadtare, R. Pasricha, and M. Sastry, "Capping of Gold Nanoparticles by the Amino Acid Lysine Renders Them Water-Dispersible," Langmuir, vol. 19, no. 8, pp. 3545-3549, Mar. 2003.

[69] D. Zare, A. Akbarzadeh, and N. Bararpour, "Synthesis and Functionalization of Gold Nanoparticles by Using of Poly Functional Amino Acids," Int. J. Nanosci. Nanotechnol., vol. 6, no. 4, pp. 223-230, Dec. 2010.

[70] M. Shu, F. He, Z. Li, X. Zhu, Y. Ma, Z. Zhou, Z. Yang, F. Gao, and M. Zeng, "Biosynthesis and Antibacterial Activity of Silver Nanoparticles Using Yeast Extract as Reducing and Capping Agents," Nanoscale Res. Lett., vol. 15, no. 14, Jan. 2020.

[71] C. Song, M. G. Blaber, G. Zhao, P. Zhang, H. C. Fry, G. C. Schatz, and N. L. Rosi, "Tailorable plasmonic circular dichroism properties of helical nanoparticle superstructures," Nano Lett., vol. 13, no. 7 , pp. 3256-3261, June 2013.

[72] K. Esashika, R. Ishii, S. Tokihiro, and T. Saiki, "Simple and rapid method for homogeneous dimer formation of gold nanoparticles in a bulk suspension based on van der Waals interactions between alkyl chains," Opt. Mater. Express, vol. 9, no. 4, pp. 1667-1677, Apr. 2019.

[73] S. R. Johnson, S. D. Evans, and R. Brydson, "Influence of a Terminal Functionality on the Physical Properties of Surfactant-Stabilized Gold Nanoparticles," Langmuir, vol. 14, no. 23, pp. 6639-6647, Oct. 1998.

[74] J. Zong, S. L. Cobb, and N. R. Cameron, "Peptidefunctionalized gold nanoparticles: versatile biomaterials for diagnostic and therapeutic applications," Biomater. Sci., vol. 5, no. 5, pp. 872-886, Mar. 2017. 


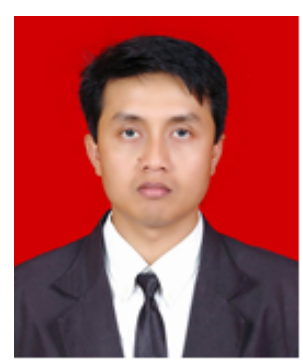

Wahyudiono received his doctoral degree in the field of Material and Life Science from Kumamoto University in 2007. He was a Research Associate of Global COE from 2008 to 2009 and a JSPS postdoctoral fellow from 2009 to 2011 in Kumamoto University, Japan. Currently, he is an Assistant Professor in Nagoya University, Japan. His main research interests are reaction engineering and material processing using sub- and supercritical fluids, electrospinning, and plasma process. More than 140 articles and 6 chapters books have been published in international peer-reviewed journals.

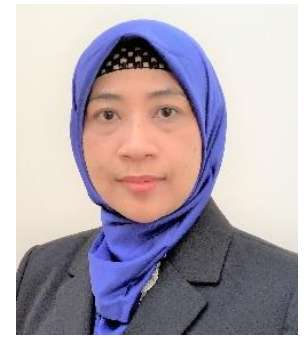

Siti Machmudah completed her doctoral degree at the Graduate School of Science and Technology, Kumamoto University, Japan in 2008. She was a postdoctoral researcher from 2008 to 2009, and a JSPS postdoctoral fellow from 2009 to 2011 in Kumamoto University, Japan. Currently, she is a senior lecturer in Department of Chemical Engineering and a Director of Directorate of Education in Institut Teknologi Sepuluh Nopember (ITS), Surabaya, Indonesia. She is also a visiting associate professor in Nagoya University. She serves as Associate Editor of Engineering Journal. Her main research interests are separation engineering, reaction engineering, and material processing using sub- and supercritical fluids.

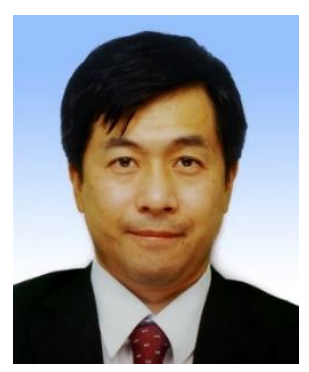

Hideki Kanda received his Ph.D. in Chemical Engineering from Kyoto University in 2000. He then worked at the Central Research Institute of Electric Power Industry, a joint subsidiary of nine Japanese electric power companies. 2008, he was awarded the Advanced Technology Award in the presence of the Japanese imperial family. 2011, he was designated as a PRESTO researcher by the Japan Science and Technology Agency, and turned to academic research. 2012, he became an assistant professor at Nagoya University. His previous research includes coal-fired power plant, molecular simulations related to adsorption, high-pressure fluid extraction, and plasma technologies.

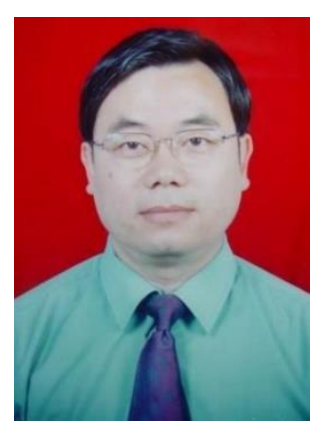

Yaping Zhao is a Professor at the School of Chemistry and Chemical Engineering at Shanghai Jiao Tong University in China. His major research focuses on the fundamental and applied aspects of Chemistry and Process Engineering in Supercritical Fluids, with applications related to nanomaterials, two-dimensional materials, polymeric foams, and silica aerogels. He has published more than 130 articles and holds more than 20 patents. He has set up a series of commercial plants for extracting bioactive and natural ingredients for pharmaceutical and food applications and has developed a green technique to produce graphene and other twodimensional materials using supercritical carbon dioxide. In 2015, he was selected as an entrepreneurship and innovation talent in Jiangsu Province, China. He is an associate Editor of The Journal of Supercritical Fluids.

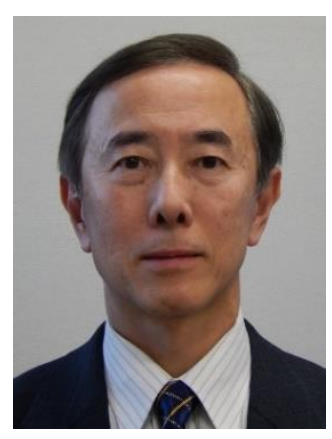

Motonobu Goto obtained BS, MSc, and Dr. Eng. from Nagoya University, Japan. He worked at Kumamoto University, Japan and moved to Nagoya University, Japan in 2012. He is actively involved in researches in supercritical fluid technology and plasma technology. He has published more than 400 papers in international journals. 